\title{
Hypospadias-intellectual disability, Goldblatt type syndrome
}

INSERM

\section{Source}

INSERM. (1999). Orphanet: an online rare disease and orphan drug data base.

Hypospadias-intellectual disability, Goldblatt type syndrome. ORPHA:2261

Hypospasdias intellectual deficit, Goldblatt type is a very rare multiple congenital anomalies syndrome described in three brothers of one South-African family, and characterized by hypospadias and intellectual deficit, in association with mirocephaly, craniofacial dysmorphism, joint laxity and beaked nails. 